\title{
Ethane in Titan's Stratosphere from Cassini CIRS Far- and Mid-infrared Spectra
}

\author{
Nicholas A. Lombardo ${ }^{1,2}$, Conor A. Nixon ${ }^{2}$ (1) Melody Sylvestre $^{3}$, Donald E. Jennings ${ }^{2}$, Nicholas Teanby ${ }^{3}$ (1), \\ Patrick J. G. Irwin ${ }^{4}$, , and F. Michael Flasar ${ }^{2}$ \\ ${ }^{1}$ Center for Space Science and Technology, University of Maryland, Baltimore County, 1000 Hilltop Circle, Baltimore, MD, USA; nicholas.lombardo@yale.edu \\ ${ }^{2}$ Goddard Space Flight Center, 8800 Greenbelt Road, Greenbelt, MD, 20770, USA \\ ${ }^{3}$ School of Earth Sciences, University of Bristol, Wills Memorial Building, Queens Road, Bristol, BS8 1RJ, UK \\ ${ }^{4}$ Atmospheric, Oceanic and Planetary Physics, Clarendon Laboratory, University of Oxford, Parks Road, Oxford OX1 3PU, UK \\ Received 2018 December 7; revised 2019 March 4; accepted 2019 March 6; published 2019 April 2
}

\begin{abstract}
The Cassini Composite Infrared Spectrometer (CIRS) observed thermal emission in the far- and mid-infrared (from 10 to $1500 \mathrm{~cm}^{-1}$ ), enabling spatiotemporal studies of ethane on Titan across the span of the Cassini mission from 2004 through 2017. Many previous measurements of ethane on Titan have relied on modeling the molecule's mid-infrared $\nu_{12}$ band, centered on $822 \mathrm{~cm}^{-1}$. Other bands of ethane at shorter and longer wavelengths were seen, but have not been modeled to measure ethane abundance. Spectral line lists of the far-infrared $\nu_{4}$ torsional band at $289 \mathrm{~cm}^{-1}$ and the mid-infrared $\nu_{8}$ band centered at $1468 \mathrm{~cm}^{-1}$ have recently been studied in the laboratory. We model CIRS observations of each of these bands (along with the $\nu_{12}$ band) separately and compare the retrieved mixing ratios from each spectral region. Nadir observations of the $\nu_{4}$ band probe the low stratosphere below $100 \mathrm{~km}$. Our equatorial measurements at $289 \mathrm{~cm}^{-1}$ show an abundance of $(1.0 \pm 0.4) \times 10^{-5}$ at $88 \mathrm{~km}$ from 2007 to 2017. This mixing ratio is consistent with measurements at higher altitudes, in contrast to the depletion that many photochemical models predict. Measurements from the $\nu_{12}$ and $\nu_{8}$ bands are comparable to each other, with the $\nu_{12}$ band probing an altitude range that extends deeper in the atmosphere. We suggest that future studies of planetary atmospheres may observe the $\nu_{8}$ band, enabling shorter wavelength studies of ethane. There may also be an advantage to observing both the ethane $\nu_{8}$ band and nearby methane $\nu_{4}$ band in the same spectral window.
\end{abstract}

Key words: infrared: planetary systems - planets and satellites: atmospheres - planets and satellites: individual (Titan)

\section{Introduction}

The largest moon of Saturn, Titan, is unique among moons in the solar system for having a substantial atmosphere. The two primary constituents are molecular nitrogen $\left(\mathrm{N}_{2}\right.$, comprising between $95 \%$ and $99 \%$ by volume) and methane $\left(\mathrm{CH}_{4}, 1.1 \%-\right.$ $1.5 \%$ as measured in Lellouch et al. 2014). Photodissociation of the two primary species give rise to molecular hydrogen $\left(\mathrm{H}_{2}\right.$, 0.1\%-0.3\%; Cui et al. 2009; Niemann et al. 2010), carbon monoxide (CO, $50 \mathrm{ppm}$ uniformly mixed; Rengel et al. 2014), ethane $\left(\mathrm{C}_{2} \mathrm{H}_{6}\right.$, about $10 \mathrm{ppm}$ in the lower stratosphere; Vinatier et al. 2015), and many larger hydrocarbons and nitriles.

The first band of ethane detected on Titan was the $\nu_{12}$ band centered at $822 \mathrm{~cm}^{-1}$ using the University of California, San Diego-University of Minnesota $60 \mathrm{inch}(152 \mathrm{~cm})$ telescope located at the Mount Lemmon Observing Facility by Gillett et al. (1973), though it was not definitively attributed to ethane until Danielson et al. (1973). Using spatially resolved observations from the Infrared Interferometer Spectrometer (IRIS) instrument on the Voyager 1 spacecraft, which made a flyby of Titan in 1981, Coustenis et al. (1989) searched for vertical and longitudinal variations in Titan's stratosphere, and concluded that the molecule was well mixed with a volume mixing ratio (VMR) of $1.3_{-0.7}^{+0.5} \times 10^{-5}$, consistent with today's measurements. High spatial resolution observations from the Cassini spacecraft enabled the study of seasonal, meridional (latitudinal), and vertical variations in the abundance of ethane, which have been included in photochemical and dynamical models of Titan. Radar observations and theoretical modeling of the Ontario Lacus and Ligeia Mare lakes on Titan have shown their composition to be up to $40 \%$ ethane, however the molecule was not detected in Punga Mare or Buffin Sinus
(Mastrogiuseppe et al. 2017, 2018, see also Cordier et al. 2009, 2012). In the stratosphere, between 100 and $500 \mathrm{~km}$, ethane has a roughly constant abundance of $10^{-5}$, as measured by the Composite Infrared Spectrometer (CIRS; Vinatier et al. 2007, 2015). In situ measurements of Titan's thermosphere near $1000 \mathrm{~km}$ by the Ion and Neutral Mass Spectrometer also on Cassini have shown ethane to have an abundance of about $4.5 \times 10^{-5}$ (Magee et al. 2009). The abundance of ethane between the surface and $100 \mathrm{~km}$ is not well known. At the time of this study, accurate measurements of ethane from the Huygens Gas Chromatograph and Mass Spectrometer have not been reported, and only approximate abundances with large uncertainties are given in Niemann et al. (2010). The team reports an ethane abundance at $88 \mathrm{~km}$ between $10^{-6}$ and $1.2 \times 10^{-5}$, over an order of magnitude in range. Radiative transfer modeling of ground-based and CIRS spectra have also been unable to probe below about $100 \mathrm{~km}$ due to the high opacity of available spectral bands. Even if nadir observations are used, thermal emission from this band generally originates from altitudes above $100 \mathrm{~km}$ (Coustenis et al. 2010).

Shortly after the arrival of the Cassini spacecraft to the Saturn system, Griffith et al. (2006) discovered ethane clouds at latitudes poleward of $50^{\circ} \mathrm{N}$ at altitudes between 30 and $50 \mathrm{~km}$. Discovered while the north pole was in winter, the clouds evolved and eventually dissipated as Titan entered equinox (LeMouélic et al. 2012).

In the mid-infrared, between about 600 and $3000 \mathrm{~cm}^{-1}(16.7$ and $3.3 \mu \mathrm{m}$ ), ethane has several infrared-active rovibrational bands including the $\nu_{1}, \nu_{5}, \nu_{7}$, and $\nu_{10}$ bands between 2700 and $3100 \mathrm{~cm}^{-1}$, the $\nu_{2}, \nu_{8}, \nu_{6}$, and $\nu_{11}$ bands between 1350 and $1600 \mathrm{~cm}^{-1}$, and the $\nu_{12}$ band centered at $822 \mathrm{~cm}^{-1}$. Of these 


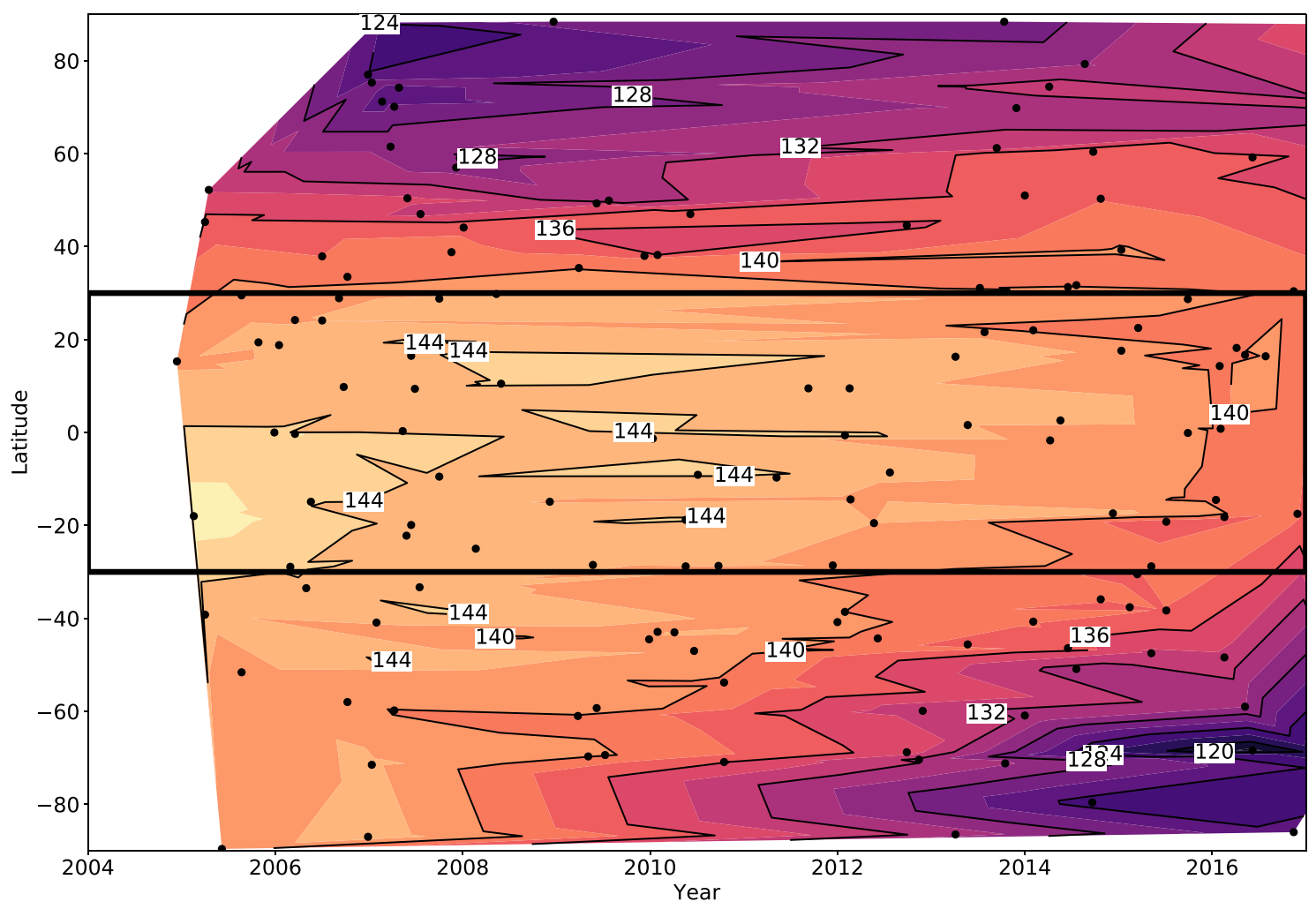

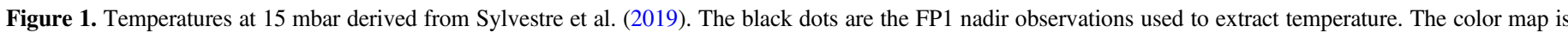
interpolated from the temperatures of individual observations. The boundary of the averaging bin is shown as the black box.

bands, the strong $\nu_{12}$ band has been used the most to model Titan's atmospheric spectra and extract the ethane abundance between 100 and $500 \mathrm{~km}$ (Vinatier et al. 2007, 2015).

Previous attempts to retrieve the ethane abundance from the $\nu_{6}$ and $\nu_{8}$ bands between 1350 and $1500 \mathrm{~cm}^{-1}$ have proved to be difficult. In particular, Coustenis et al. (2010) found it difficult to extract precise abundances of ethane from this region potentially due to a lack of $\mathrm{C}_{3} \mathrm{H}_{8}$ line data at the time and inaccurate line data for $\mathrm{C}_{2} \mathrm{H}_{6}$. Since then, new line data for $\mathrm{C}_{2} \mathrm{H}_{6}$ and $\mathrm{C}_{3} \mathrm{H}_{8}$ have been made available, allowing our analysis to proceed (di Lauro et al. 2012; Sung et al. 2013).

In the far-infrared, ethane has a single mode, the $\nu_{4}$ torsional band centered at $289 \mathrm{~cm}^{-1}$. Until recently, spectral line lists for this band were not available, and thus retrievals utilizing this band were not possible. However, lab work presented in Moazzen-Ahmadi et al. (2015) has enabled the creation of a spectral line list for this band of ethane. The $\nu_{4}$ band is less intense than the $\nu_{12}$ band, thus nadir observations are able to probe deeper through Titan's atmosphere before optical saturation. Therefore, deeper soundings of ethane abundance on Titan are now possible by modeling the $\nu_{4}$ band seen in nadir observations of Titan's disk, described in this paper for the first time.

\section{Methods}

The CIRS was a Fourier-transform infrared spectrometer on the Cassini spacecraft that explored the Saturn system from 2004 until its intentional deorbit into Saturn in 2017 September. CIRS had three focal planes, each sensitive to a different region of the infrared spectrum. Focal plane 1 (FP1) was sensitive in the far-infrared from 10 to $600 \mathrm{~cm}^{-1}$, focal plane 3 (FP3) was sensitive in the mid-infrared from 600 to $1100 \mathrm{~cm}^{-1}$, and focal plane 4 (FP4) was sensitive in the midinfrared from 1100 to $1500 \mathrm{~cm}^{-1}$ (Jennings et al. 2017).

\subsection{Far-infrared Dataset}

FP1 comprised a single detector, with a field of view of about $4 \mathrm{mrad}$. Nadir observations in this study were performed at a spectral resolution of $0.5 \mathrm{~cm}^{-1}$ at emission angles between $45^{\circ}$ and $85^{\circ}$, averaged over the footprint of the detector, at a range from $1.5 \times 10^{5}$ to $3.5 \times 10^{5} \mathrm{~km}$ from Titan. At these distances, the size of the detector footprint on the surface of Titan varies between 600 and $1400 \mathrm{~km}$. Data observed at high emission angles will have traveled a longer path length though the atmosphere, thus increasing the signal-to-noise ratio $(\mathrm{S} / \mathrm{N})$ of the $\nu_{4}$ ethane emission. Though data observed at lower emission angles will have traveled a shorter path length, the ethane emission will have originated from the same source altitude.

To increase the $\mathrm{S} / \mathrm{N}$ of trace gases in the spectra we modeled, we combined observations between $30^{\circ} \mathrm{S}$ and $30^{\circ} \mathrm{N}$ from 2007 through 2017. These latitude bounds were chosen because, as shown in Figure 1, Titan's temperature at the altitude where our model is sensitive to (about $88 \mathrm{~km}$ ) is similar over the duration of the mission. This time range was chosen as it includes most of the data taken by CIRS in this latitude region, while also excluding a period of time in 2007 where a large noise feature was present in the FP1 spectra. This average includes data from 6624 spectra.

A map of the temperatures at $15 \mathrm{mbar}$ (about $88 \mathrm{~km}$, where our model is sensitive to) is shown in Figure 1. Temperatures 
Table 1

Table of Ethane Bands Modeled in This Study, as Well as a Summary of the Spectral Bins Used and Altitude Sensitivity of the Retrievals

\begin{tabular}{lcccc}
\hline \hline Band & Band Center & Wavenumbers Modeled & Observation Type & Altitude Sensitivity \\
\hline$\nu_{4}$ & $289 \mathrm{~cm}^{-1}$ & $240-300 \mathrm{~cm}^{-1}$ & FP1 Nadir & $88 \mathrm{~km}$ \\
$\nu_{12}$ & $822 \mathrm{~cm}^{-1}$ & $800-860 \mathrm{~cm}^{-1}$ & FP3 Limb & $152-380 \mathrm{~km}$ \\
$\nu_{8}$ & $1468 \mathrm{~cm}^{-1}$ & $1440-1480 \mathrm{~cm}^{-1}$ & FP4 Limb & $212-373 \mathrm{~km}$ \\
\hline
\end{tabular}

Note. The altitude sensitivity lists only the altitude of the maximum contribution for each bin, and does not include the FWHM of the contribution function. We do not make use of mid-infrared nadir observations, as previous studies have shown that nadir observations of the $\nu_{12}$ band only probe to about $100 \mathrm{~km}$ (Bampasidis et al. 2012).

are from Sylvestre et al. (2019), and are calculated by modeling the $\mathrm{CH}_{4}$ rotational lines in FP1.

\subsection{Mid-infrared Dataset}

FP3 and FP4 were both linear arrays of 10 detectors, with a field of view of about $0.27 \mathrm{mrad}$ per detector. The smaller fields of view of FP3 and FP4 allowed for vertically resolved observations of Titan's limb and vertical profiles of molecular abundances to be measured. In the observations used in this study, the centers of the fields of view of the FP3 and FP4 arrays were positioned normal to Titan's surface at two tangent altitudes of 125 and $225 \mathrm{~km}$. Limb observations in this study were performed at a spectral resolution of $0.5 \mathrm{~cm}^{-1}$. Spectra were vertically binned into non-overlapping $50 \mathrm{~km}$ bins, from 100 to $400 \mathrm{~km}$. To enable a proper comparison to our FP1 measurements, we make use of the same time-latitude averaging scheme as in our FP1 analysis.

In FP3, we model the $\mathrm{C}_{2} \mathrm{H}_{6} \nu_{12}$ band which has been modeled extensively in previous literature. In FP4 we model the $\nu_{8}$ band centered at $1468 \mathrm{~cm}^{-1}$. Also present in FP4 is the $\nu_{6}$ band centered at $1379 \mathrm{~cm}^{-1}$. We included this band in our modeling, however results derived from this band were noisy and unreliable, due to the presence of stronger methane and propane emissions.

A summary of the bands modeled in this study is shown in Table 1.

\subsection{Spectral Line Data}

Spectral line data used in the modeling of all three focal planes were taken from the 2016 high-resolution transmission molecular absorption (HITRAN) database (Gordon et al. 2017), which includes several bands of ethane, including the $\nu_{12}$ band, $\nu_{4}$ band (first studied in Moazzen-Ahmadi et al. 2015), and the $\nu_{6}$ and $\nu_{8}$ bands (originally reported in di Lauro et al. 2012). Also from HITRAN are line data for $\mathrm{H}_{2} \mathrm{O}$ and $\mathrm{CH}_{4}$. Line data for $\mathrm{C}_{2} \mathrm{~N}_{2}$, and $\mathrm{C}_{3} \mathrm{H}_{4}$, not available in HITRAN, were taken from the GEISA database (Jacquinet-Husson et al. 2016). Line data for propane is not available in HITRAN, so our modeling makes use of the pseudo-line list presented in Sung et al. (2013).

\subsection{Radiative Transfer Modeling}

Spectral modeling was performed with the non-linear optimal estimator for multivariate spectral analysis (NEMESIS) inverse radiative transfer code (Irwin et al. 2008). NEMESIS operates on the method of optimal estimation, which involves the computation of a forward model and a retrieval process. Forward models were calculated with the correlated-k method described in Lacis \& Oinas (1991). To recreate the instrumental line shape, the model includes a Hamming apodization of a FWHM of $0.475 \mathrm{~cm}^{-1}$. The retrieval process operates by varying user-defined a priori profiles of chosen physical parameters and assumed uncertainties (such as temperature, aerosol abundance, and trace gas VMRs) to optimize the spectral fits. It is important to define a realistic a priori profile and uncertainty, as providing unrealistic conditions will yield unrealistic results. For example, defining the a priori error too small on a gas profile that is being retrieved can over-constrain the model; the reader is directed to Irwin et al. (2008) for more information. Optimization of the fit of the synthetic spectrum to the measured spectrum is performed by minimizing the cost function, a parameter that includes the deviation of the retrieved profile from the a priori estimate, and the quality of fit to the spectra (similar to a $\chi^{2}$ goodness-of-fit test). NEMESIS has been extensively used to determine atmospheric abundances in the outer solar system using infrared spectra, and the application to Titan is described in Teanby et al. (2009), Cottini et al. (2012), Sylvestre et al. (2018), and Lombardo et al. (2019) and references therein.

In FP1, we model the spectral window from 240 to $300 \mathrm{~cm}^{-1}$. A temperature profile derived from a weighted average of temperatures reported in Sylvestre et al. (2019) was used. Trace gas features in this region include $\mathrm{H}_{2} \mathrm{O}$ at $253 \mathrm{~cm}^{-1}, \mathrm{C}_{2} \mathrm{~N}_{2}$ centered at $233 \mathrm{~cm}^{-1}$ with emission from weak lines noticeable at wavenumbers less than $250 \mathrm{~cm}^{-1}$ and more prominent in the winter hemisphere, and $\mathrm{C}_{2} \mathrm{H}_{6}$ at $289 \mathrm{~cm}^{-1}$. Also contributing to the spectral radiance in this region is $\mathrm{H}_{2}$ which contributes to the collision-induced absorption and an aerosol haze emission that contributes to the continuum. Over the small spectral window that we model, we assume that aerosol haze has a gray spectral dependence (or spectrally flat), consistent with aerosol spectra retrieved in Anderson \& Samuelson (2011). $\mathrm{C}_{2} \mathrm{H}_{6}, \mathrm{C}_{2} \mathrm{~N}_{2}$, and the aerosol haze are allowed to vary with altitude in the retrieval process, while $\mathrm{H}_{2}$ is allowed to vary as a constant-with-altitude profile. $\mathrm{H}_{2} \mathrm{O}$ is set at a constant abundance of $2 \times 10^{-10}$, consistent with Cottini et al. (2012). At slightly higher wavenumbers, $\mathrm{C}_{3} \mathrm{H}_{4}$ contributes strongly to the spectral radiance, however contribution at wavenumbers less than $300 \mathrm{~cm}^{-1}$ is negligible. Further discussion of modeling the continuum in this region is discussed in the following subsection.

For the mid-infrared modeling, we first retrieve a mean temperature profile for the $30^{\circ} \mathrm{S}-30^{\circ} \mathrm{N}$ latitude bin for the time range covered by the mid-infrared observations. We model the $1300-1380 \mathrm{~cm}^{-1}$ region of FP4, including the $\mathrm{Q}$ and $\mathrm{R}$ branches of the $\nu_{4}$ band of methane. Our retrieval process involves setting a constant methane abundance of $1.1 \%$ above the saturation altitude, as measured in Lellouch et al. (2014) and allowing a temperature a priori profile derived from a weighted average of temperatures presented in Achterberg et al. (2014) to vary. An aerosol haze contributing to the continuum 

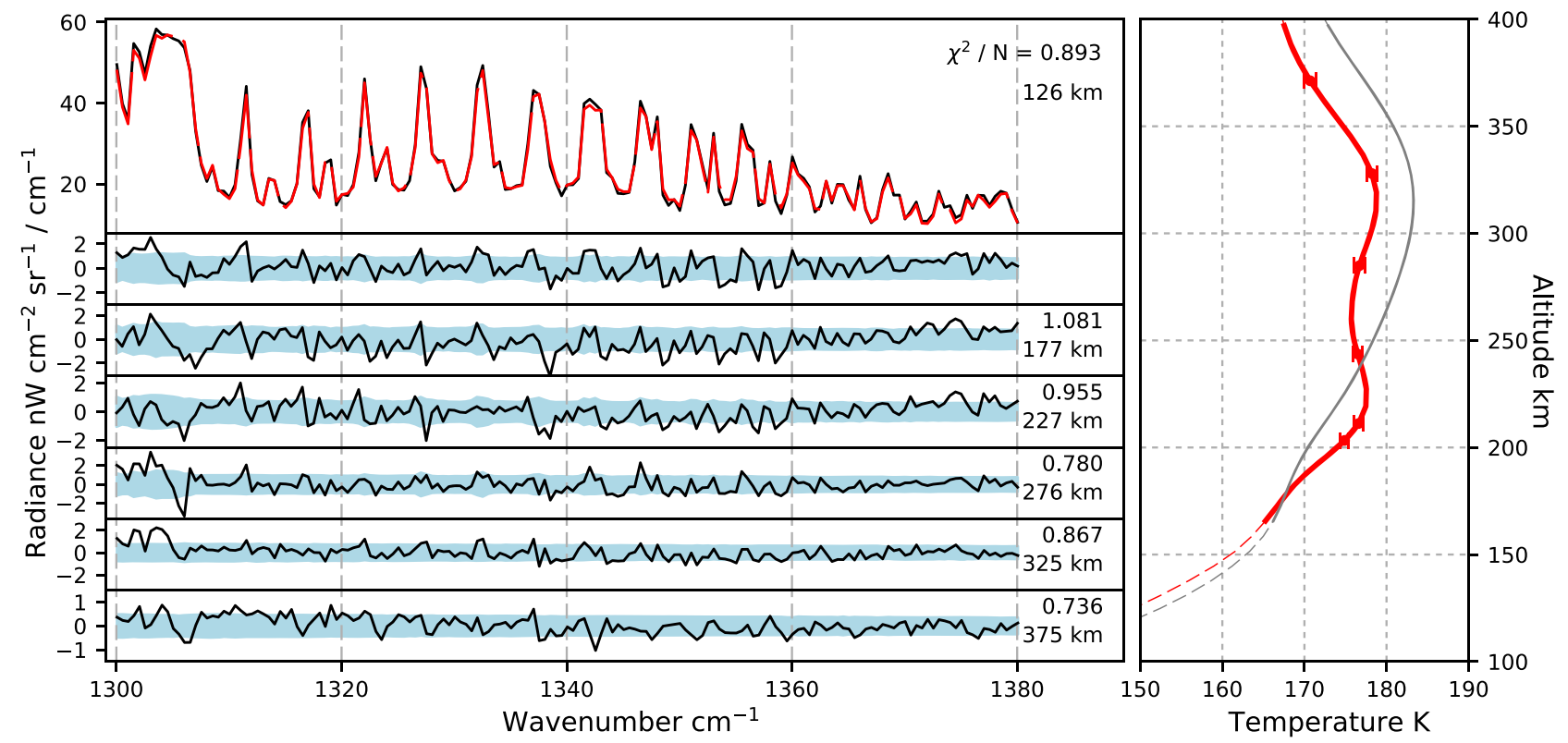

Figure 2. Model fits for the FP4 temperatures retrievals. Left: the original data (black) compared to the synthetic spectrum (red dashed) for the lowest altitude bin (topmost plot). The residuals (observed data minus synthetic spectra) are shown for all of the modeled spectra below. The blue envelopes are the model error. The values labeled on each spectrum are the modified $\chi^{2}$ value (top) and center altitude of each bin (bottom). Right: the retrieved temperature profile (red) and error bars shown with the a priori (gray) profile used. The a priori is a weighted average of the temperatures presented in Achterberg et al. (2014).
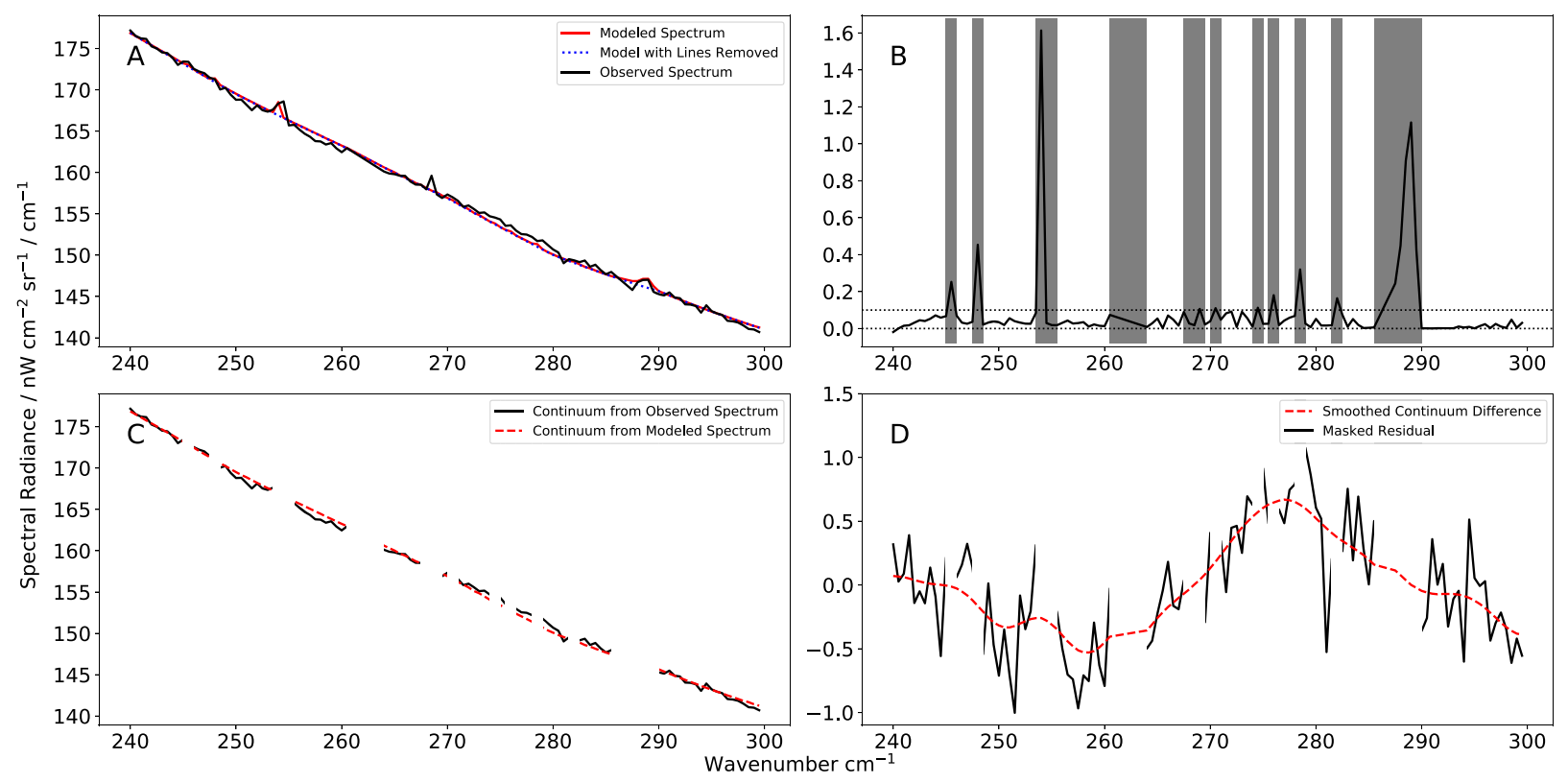

Figure 3. (A) Observed spectrum (black), modeled spectrum (red), and modeled spectrum with molecular lines removed (black dotted). The unmodeled continuum variation can be seen roughly between 270 and $290 \mathrm{~cm}^{-1}$. (B) The contribution from molecular lines, calculated by subtracting the model calculated with no molecular lines from the modeled spectrum. Wavenumbers where the gas contribution is above $0.1 \mathrm{nW}$ are masked to modify only the continuum and are highlighted by gray boxes in the figure. (C) The continuum (region where molecular contribution is minimal) from the model compared to the observations. (D) The difference between the observed and modeled continua (black). The red dashed line is the smoothed and used as the spectral dependence for a second aerosol haze.

of the spectra is also allowed to vary with altitude in the retrieval process. The modeled spectra and mean temperature profile used in our mid-infrared modeling is shown in Figure 2.

In FP3, we model the spectral window from 800 to $860 \mathrm{~cm}^{-1}$. This region is dominated by the $\nu_{12}$ band of ethane, however the $\nu_{8}$ band of propane (see Nixon et al. 2009), centered at $869 \mathrm{~cm}^{-1}$, may contribute to the continuum in the higher wavenumber end of this region. In addition to these molecules, we also include a non-gray haze to model the continuum. The opacity of the haze increases linearly with wavenumber over this region.

In FP4, we model a spectral window from 1440 to $1480 \mathrm{~cm}^{-1}$ including the $\nu_{8}$ band centered at $1468 \mathrm{~cm}^{-1}$. This window includes the $\nu_{17}$ and $\nu_{24}$ bands of propane, of which only the $\nu_{24}$ Q-branch at $1472 \mathrm{~cm}^{-1}$ is noticeable (Figure 8). We also include a non-gray haze to model the continuum in this region. A gray haze does not accurately model the continuum in the higher 


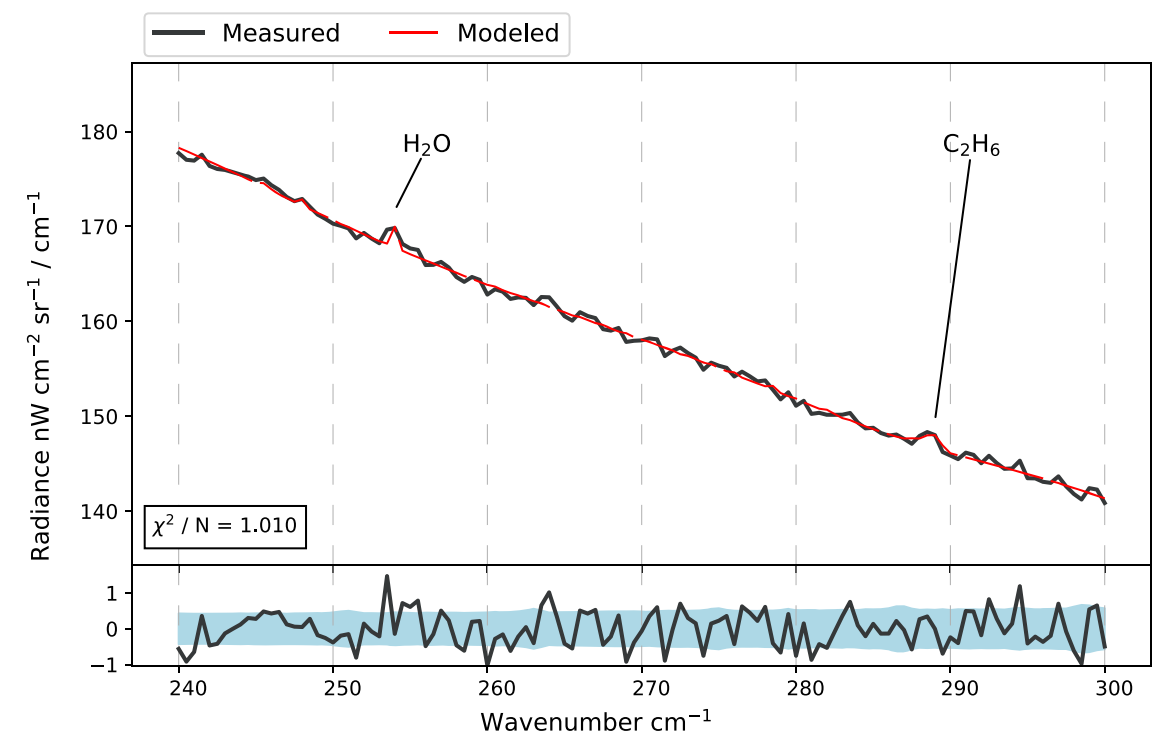

Figure 4. Upper panel: synthetic spectrum (red) compared with the observed spectrum (black) of the $\nu_{4}$ band. Lower panel: the residual of the fit (black), or the synthetic spectrum minus the observed spectrum, and $1 \sigma$ error envelope (light blue). Where the residual is positive, the model is dimmer than the observation, and where the residual is negative, the model is brighter than the observation. When the continuum is modeled with the previously described method, we are able to accurately model this spectral region. The contribution from ethane to the spectrum can be seen as a small emission in the spectrum, which we can fit well.

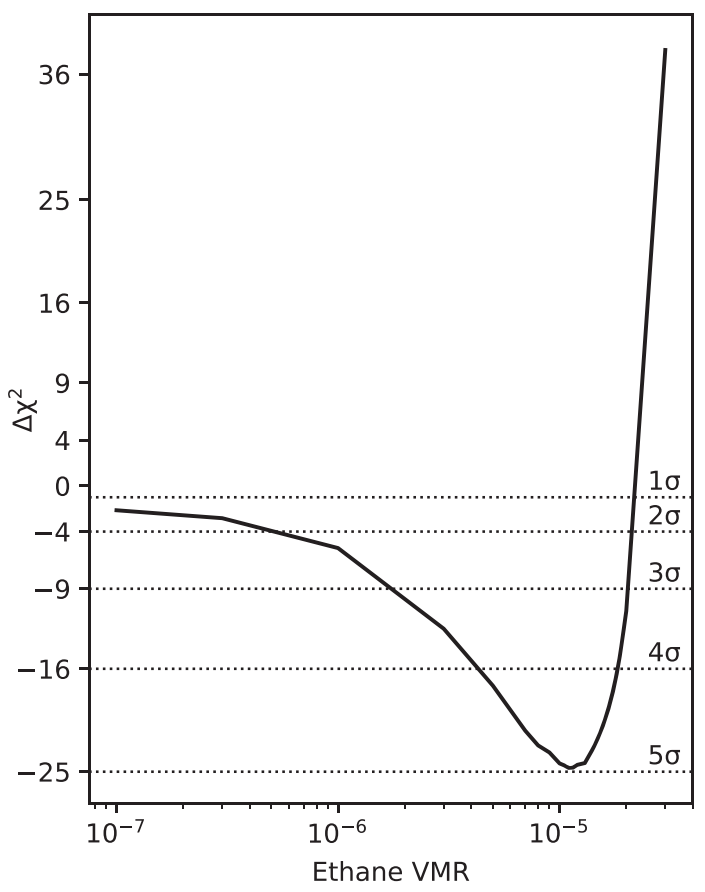

Figure 5. $\Delta \chi^{2}$ values plotted against the ethane abundance in the forward model. The $\Delta \chi^{2}$ value achieves a minimum of -25 at $1.1 \times 10^{-5}$, consistent with the retrieved measurement.

wavenumbers of FP4, so we include a haze spectral response derived from Vinatier et al. (2012).

\subsubsection{Unidentified Far-infrared Continuum Feature}

While only including spectral characteristics of $\mathrm{H}_{2} \mathrm{O}, \mathrm{C}_{2} \mathrm{~N}_{2}$, $\mathrm{C}_{2} \mathrm{H}_{6}, \mathrm{H}_{2}$, and a gray aerosol, we noticed a prominent emission feature in the residual of the synthetic spectrum (Figure 3 ). The feature is broad, spanning the region between 270 and $290 \mathrm{~cm}^{-1}$, and appears in most bins. To model this feature, we take an approach similar to that of Teanby et al. (2013).

First, we perform a retrieval as previously described, allowing the aforementioned parameters to vary. Then, we remove spectral line data from $\mathrm{H}_{2} \mathrm{O}, \mathrm{C}_{2} \mathrm{~N}_{2}$, and $\mathrm{C}_{2} \mathrm{H}_{6}$, and using the retrieved haze and $\mathrm{H}_{2}$ abundance, run a forward model. This allows us to examine only the continuum of the modeled spectrum (Figure 3(A)). We then subtract this forward modeled continuum from the synthetic spectrum to identify the contribution from molecular lines (Figure 3(B)). We then mask regions where this difference exceeds $0.1 \mathrm{nW} \mathrm{cm}{ }^{-2} \mathrm{sr}^{-1} \mathrm{~cm}^{-1}$, so that we identify wavenumbers that show only continuum. The difference between the masked retrieved and masked forward modeled residuals is then smoothed and set as the extinction cross section for the haze representing the unidentified feature (Figures 3(C) and (D)). The cause of this feature could be a small-scale spectral structure in the haze that is only apparent in this high-S/N large spectral average.

\section{Results}

\subsection{Focal Plane 1}

We compare the synthetic spectra to the observed spectra in Figure 4. The quality of the fit can be assessed with the $\chi^{2} / N$ value, which can be defined as

$$
\chi^{2} / N=\frac{1}{N} \sum_{\nu}\left(\frac{M(\nu)-O(\nu)}{\sigma(\nu)}\right)^{2},
$$

where $M$ is the modeled spectrum, $O$ is the observed spectrum, $\sigma$ is the noise on the observed data, and $N$ is the number of independent data points in the spectrum. A synthetic spectrum with a $\chi^{2} / N$ of 1 is then the best possible fit to the observations.

The $\mathrm{C}_{2} \mathrm{H}_{6}$ mixing ratio determined at $88 \mathrm{~km}$ (see Figure 7) from the FP1 analysis is $(1.0 \pm 0.4) \times 10^{-5}$, very close to our a priori value of $(1.0 \pm 0.5) \times 10^{-5}$. To check the sensitivity of the $\nu_{4}$ band to the a priori, we also modeled the FP1 spectral window using two additional a priori of $(3.0 \pm 1.5) \times 10^{-5}$ and $(5.0 \pm 2.5) \times 10^{-6}$. The higher a priori retrieved an 


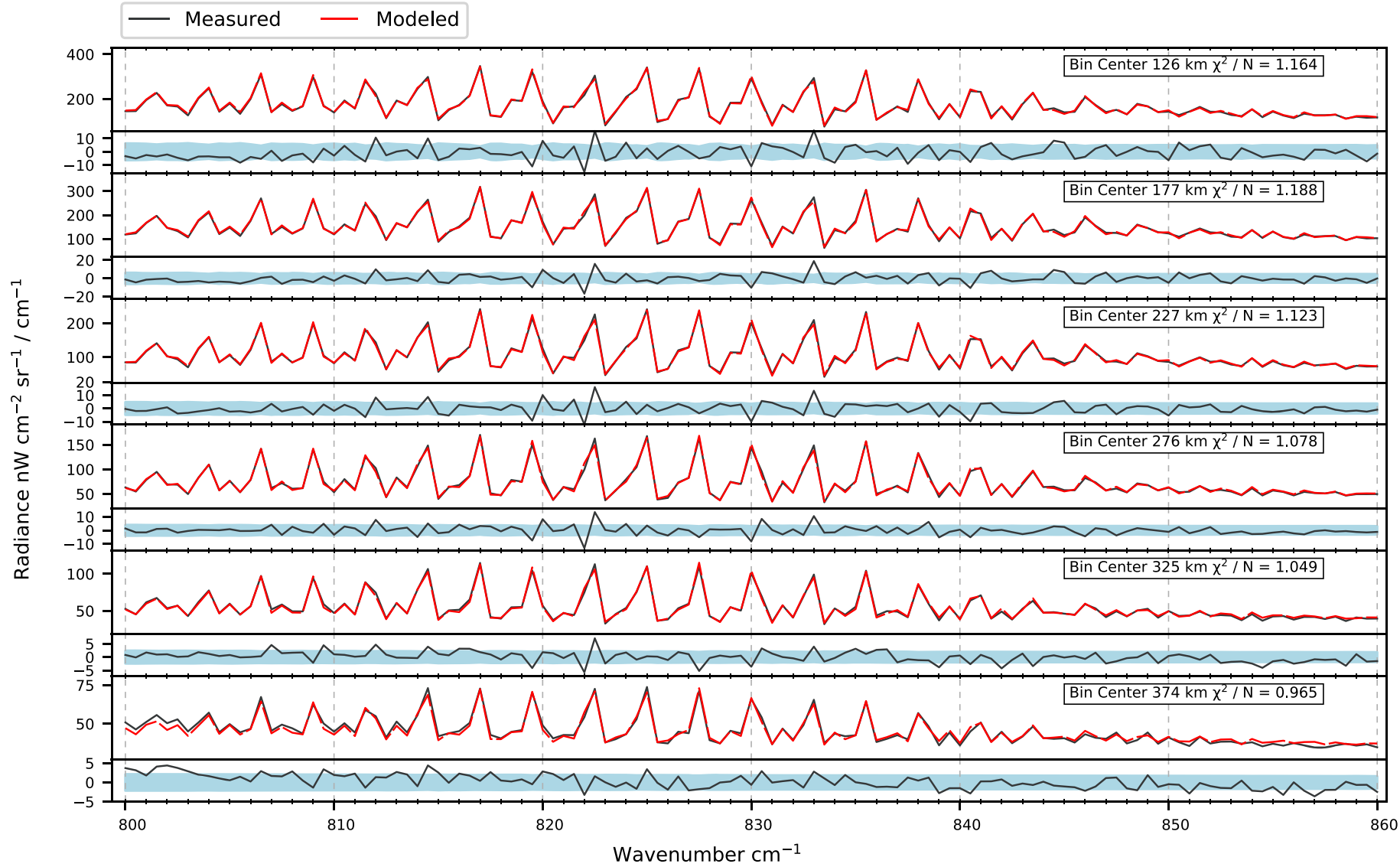

Figure 6. Same layout as that in Figure 3. The $\nu_{12}$ band is modeled well. The few peaks seen in the residual are from electrical interference in the instrument.

ethane abundance of $(1.2 \pm 0.4) \times 10^{-5}$, and the lower a priori retrieved an abundance of $(5.6 \pm 1.4) \times 10^{-6}$, both sensitive to the same altitude. The abundances determined from each a priori are within the model uncertainties, therefore we use the $10^{-5}$ a priori as it is in agreement with our $\chi^{2}$ analysis described in Section 3.1.1.

The contribution function, or the rate of change of spectral radiance with respect to the amount of the molecule included in the model, is a measure of where the model is sensitive for a certain molecule. In Figure 7, we show the contribution function for ethane retrieved in the $30^{\circ} \mathrm{S}$ to $30^{\circ} \mathrm{N}$ bin, centered at $289 \mathrm{~cm}^{-1}$. The nadir contribution function obtains a maximum value at $13.1 \mathrm{mbar}$, or an altitude between 85.7 and $87.5 \mathrm{~km}$. The FWHM of the contribution function extends from 28 to 3.7 mbar.

\subsection{1. $\chi^{2}$ Analysis}

In addition to the full retrieval already discussed, we performed a $\Delta \chi^{2}$ analysis. In this analysis, similar to that presented in Nixon et al. (2010) and Lombardo et al. (2019), we first perform a retrieval on the spectra including all molecules and aerosols previously described with the exception of ethane. The $\chi^{2}$ of this retrieval will be referred to as $\chi_{0}^{2}$ (note that this is different from the $\chi^{2} / N$ value previously described). We then set the abundance profiles of these molecules to be the retrieved values, and run forward models with varying amounts of ethane added. The ethane abundance profiles used in this method are set to be a constant value until saturation, where they follow the saturation vapor pressure curves throughout the troposphere. The $\chi^{2}$ value of the fit for each of the forward model runs will be referred to as $\chi_{m}^{2}$. The $\Delta \chi^{2}$ value is then $\chi_{m}^{2}-\chi_{0}^{2}$. Where the $\Delta \chi^{2}$ achieves a minimum value, we claim this as the most probable abundance of ethane, with a confidence of $\sqrt{\Delta \chi^{2}}$. The $1 \sigma$ confidence can be described as where $\Delta \chi^{2}-\Delta \chi_{\text {minimum }}^{2}=1$. Figure 5 shows the plot of $\Delta \chi^{2}$ versus ethane abundance. Using this method, we determine an ethane abundance of $1.1_{-0.2}^{+0.3} \times 10^{-5}$, very close to the abundance determined using a $1 \times 10^{-5}$ a priori profile and full retrieval. The uncertainties on this value are the ethane VMRs such that $\Delta \chi_{\text {minimum }}^{2}-\Delta \chi_{\mathrm{vmr}}^{2}= \pm 1$. It is expected that the abundance retrieved using NEMESIS is slightly different from the abundance determined using this $\Delta \chi^{2}$ analysis since NEMESIS will vary the abundance of several molecules at once to optimize the spectral fit of the model to the data. The measurement uncertainty reported on the retrieved abundance is slightly greater due to uncertainties in the line position, line strength, temperature, and observation error that are included in the retrieval. This $\Delta \chi^{2}$ method, however, does not include these uncertainties, hence the smaller error bars.

\subsection{Focal Plane 3}

The fit of the model to the data for FP3 is shown in Figure 6, and is generally very good. The few sharp spikes in the residual are due to electrical interference from the instrument, described by Chan et al. (2015) and Jennings et al. (2017). The contribution function for each altitude bin is plotted in Figure 7, along with the contribution function from our FP1 analysis, and shows that the data are sensitive to the region 


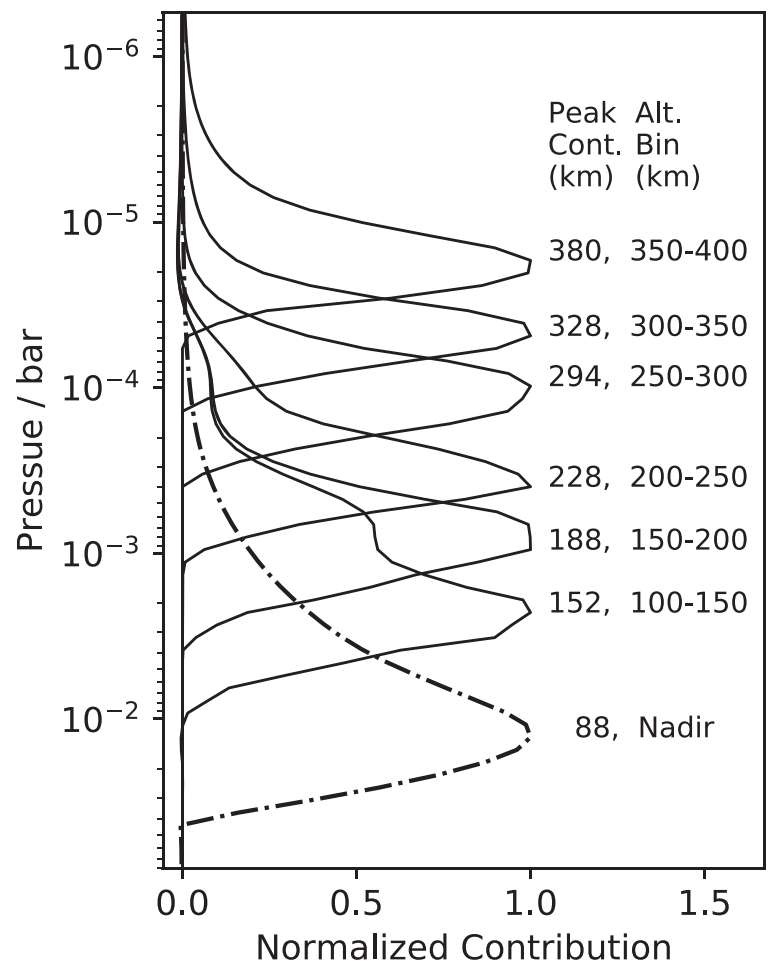

Figure 7. Normalized contribution functions at $289 \mathrm{~cm}$ for FP1 (dotteddashed) and $822 \mathrm{~cm}$ for FP3 (solid). The contribution function from FP1 clearly peaks more than $60 \mathrm{~km}$ deeper in the atmosphere than the altitude bin centered at $125 \mathrm{~km}$. The contribution function can achieve a maximum value outside of the bounds of an altitude bin if the spectrum becomes optically saturated before the model ray looking through the atmosphere reaches the tangent altitude. Though this band of ethane is optically thinner than the bands in FP4, a second source of emission can be seen originating from about $188 \mathrm{~km}$ in the lowest altitude bin being modeled, an example of the importance of the far-infrared retrieval.

between 150 and $380 \mathrm{~km}$. The retrieved vertical profile from this fit is shown in Figure 10, and is consistent with previous measurements from Vinatier et al. $(2007,2015)$.

\subsection{Focal Plane 4}

Modeling the spectral window from 1400 to $1500 \mathrm{~cm}^{-1}$ is challenging due to the number of broad features contributed by ethane, propane, methane, and other potentially undetected trace species such as butane. Additionally, aliasing from higher wavenumbers may affect the quality of the data in this region. The fit of our synthetic spectra to the observations is shown in Figure 8. The contribution functions are plotted in Figure 9, which show that even though we use the same altitude binning scheme on the limb as in our FP3 analysis, we are not sensitive to the lower altitudes. The $\nu_{8}$ band of ethane is thus optically thicker, preventing this analysis from probing lower than $200 \mathrm{~km}$. In higher altitude bins, the sensitivity of the $\nu_{8}$ band at this resolution is comparable to the sensitivity of the $\nu_{12}$ band in FP3. The retrieved profile from this model is shown in Figure 10, and is comparable to our measurements made from the FP3 spectra.

\section{Discussion}

\subsection{Comparison between Focal Planes}

The measurements made with FP3 span the region from 152 to $380 \mathrm{~km}$. The measurements made with FP4 span the region from 212 to $373 \mathrm{~km}$. The higher opacity of the $\nu_{8}$ band of $\mathrm{C}_{2} \mathrm{H}_{6}$ prevent limb sounding from probing the lower altitudes visible in the $\nu_{12}$ band. In the region from 200 to $400 \mathrm{~km}$, however, the measured $\mathrm{C}_{2} \mathrm{H}_{6}$ abundances from each band are comparable. These data are shown in Table 2.

Compared to measurements made from the $\nu_{12}$ and $\nu_{8}$ bands of ethane in FP3 and FP4, our modeling of the $\nu_{4}$ band has probed about $50 \mathrm{~km}$ deeper in Titan's stratosphere. The contribution functions of the lowest altitude bin in our FP3 and FP4 analyses achieve their maxima at altitudes of $152 \mathrm{~km}$ in FP3 and $212 \mathrm{~km}$ in FP4, $62 \mathrm{~km}$ and $124 \mathrm{~km}$ higher than the most sensitive altitude from our FP1 modeling. The mixing ratio measured at $152 \mathrm{~km}$ in our FP3 analysis is $(1.21 \pm 0.14) \times 10^{-5}$, overlapping the lower measurement at $88 \mathrm{~km}$.

\subsection{Comparison to Previous Measurements}

The measurements we made that incorporate the FP3 and FP4 data are comparable to the measurements from previous studies, including Vinatier et al. (2007, 2015), Coustenis et al. (2010), and Bampasidis et al. (2012; Figure 10). Both our measurements and the previously published measurements indicate that the mixing ratio of ethane is slowly increasing with altitude from $100 \mathrm{~km}$ through $400 \mathrm{~km}$ in Titan's stratosphere.

Searching through previous literature, the ethane measurements with the deepest sounding in Titan's stratosphere are made in Coustenis et al. (2010) and Bampasidis et al. (2012), who retrieve ethane abundance from CIRS FP3 nadir spectra. The contribution function reported in Coustenis et al. (2010) achieves a maximum at $7 \mathrm{mbar}$, roughly $100 \mathrm{~km}$. We assume that Bampasidis et al. (2012) are sensitive to the same altitude, as both groups use the same method and similar data sets. The span of mixing ratios reported in Bampasidis et al. (2012) are plotted in Figure 10 for comparison with our measurements. The vertical error on this data point is the FWHM of the contribution function for ethane reported in Coustenis et al. (2010), and the horizontal error is the span of ethane measurements, including error, reported in Bampasidis et al. (2012).

\subsection{Comparison to Photochemical Models}

To evaluate the accuracy of photochemical models, we compare our measurement at $88 \mathrm{~km}$ to predictions from Hébrard et al. (2013), Krasnopolsky (2014), Li et al. (2015), and Dobrijevic et al. (2016), plotted in Figure 11. With the exception of the model presented in Hébrard et al. (2013), which overestimates ethane throughout the stratosphere by a factor of 6 , the models we compare predict a mixing ratio about an order of magnitude lower than what we measure at $88 \mathrm{~km}$. All of the models predict that ethane is depleted in this region compared to altitudes above $100 \mathrm{~km}$. Our measurements show that at $88 \mathrm{~km}$, ethane is nearly as abundant as it is higher in the stratosphere. The discrepancy could be caused by overestimating depletion mechanisms, underestimating production mechanisms, or inaccurate eddy diffusion coefficients. We come to a similar conclusion when comparing to Vuitton et al. (2018), who predict a lower concentration of $\mathrm{C}_{2} \mathrm{H}_{6}$ throughout the stratosphere relative to our measurements. 


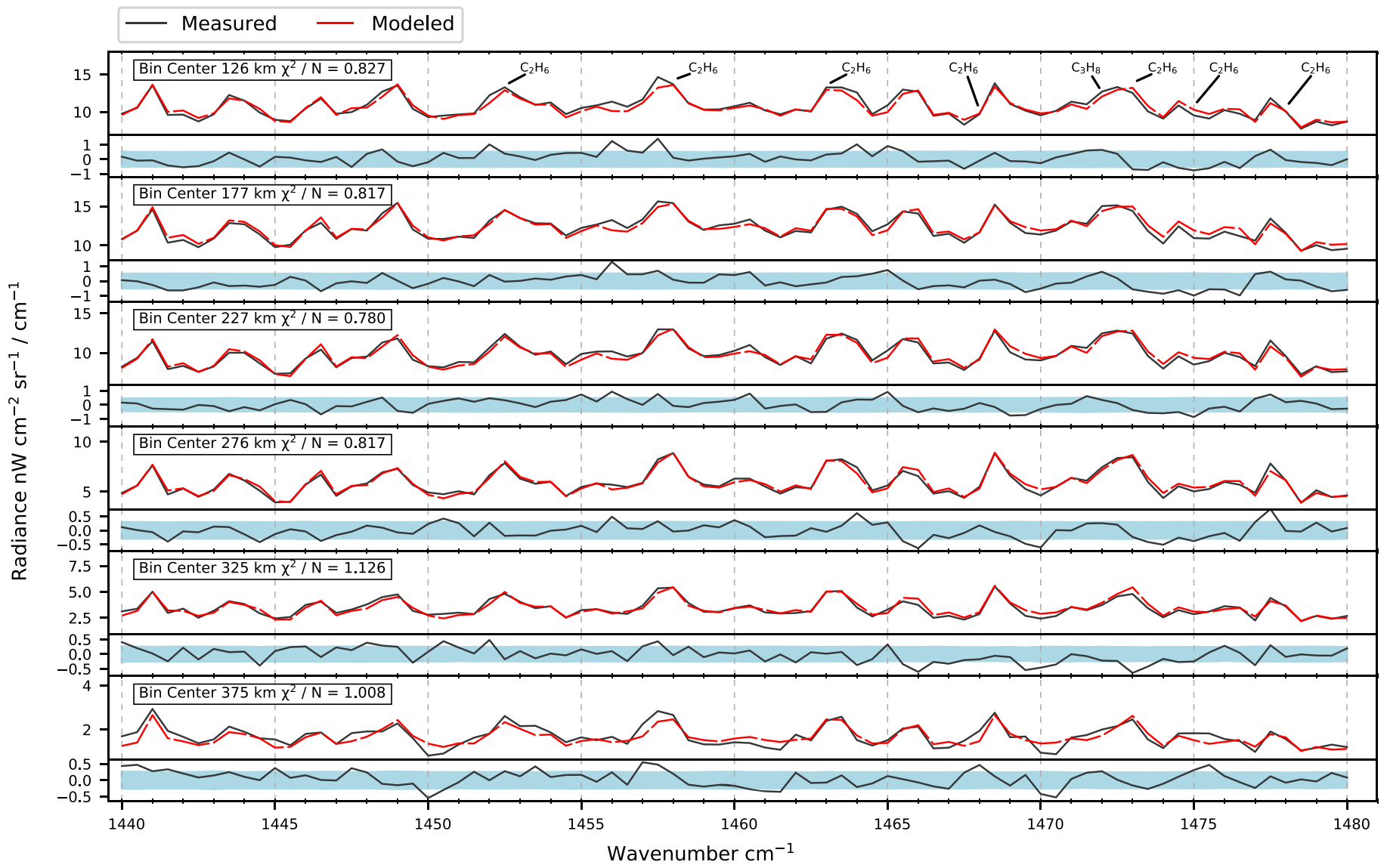

Figure 8. Same layout as that in Figure 3. The $\nu_{8}$ band is more challenging to model than the $\nu_{12}$ band because of the underlying propane band and aliasing from higher wavenumbers. The propane band centered at $1472 \mathrm{~cm}^{-1}$ may contribute to the slight discrepancy between the measurements derived from the FP3 and FP4 spectra.

Table 2

Summary of Information for Each Altitude Bin Modeled

\begin{tabular}{|c|c|c|c|c|c|c|c|c|c|c|}
\hline \multicolumn{3}{|c|}{ FP1 } & \multicolumn{4}{|c|}{ FP3 } & \multicolumn{4}{|c|}{ FP4 } \\
\hline$\ldots$ & $\ldots$ & $\ldots$ & $150-200$ & 1224 & 188 & $1.53 \pm 0.14$ & $150-200$ & 1331 & 212 & $1.42 \pm 0.19$ \\
\hline$\cdots$ & $\cdots$ & $\cdots$ & $200-250$ & 1406 & 228 & $1.62 \pm 0.14$ & $200-250$ & 1555 & 244 & $1.58 \pm 0.21$ \\
\hline$\cdots$ & $\ldots$ & $\cdots$ & $250-300$ & 1341 & 294 & $1.78 \pm 0.14$ & $250-300$ & 1491 & 286 & $1.75 \pm 0.19$ \\
\hline
\end{tabular}

Note. From left to right, the columns are the altitude bin used in the average, the number of spectra included in the average, the altitude of peak contribution for $\mathrm{C}_{2} \mathrm{H}_{6}$ at $822 \mathrm{~cm}^{-1}$ in FP3 (left) and $1458 \mathrm{~cm}^{-1}$ in FP4 (right), and the retrieved $\mathrm{C}_{2} \mathrm{H}_{6}$ abundance and uncertainty for each altitude bin.

\section{Conclusion}

Ethane is a prominent component of Titan's atmosphere, surface, and potentially subsurface. The molecule is formed high in the atmosphere and can be transported deep into the stratosphere where it may condense into clouds or precipitate onto the surface. Ethane is also expected to be a component of Titan's lakes where it may interact with the troposphere and surface of Titan. The molecule may also interact with the surface materials on Titan, potentially opening a channel to transport ethane into Titan's subsurface. To fully understand the role of ethane on Titan, we must have accurate measurements of the molecule throughout the atmosphere.

In this paper, we have measured ethane in Titan's stratosphere by modeling its $\nu_{4}$ band observed in CIRS far-infrared spectra. This marks the first time that this band has been used to measure the abundance of ethane in a planetary atmosphere. Using this optically thin band has enabled us to probe a lower altitude than permitted by the optically thicker mid-infrared $\nu_{12}$ and $\nu_{8}$ bands. 


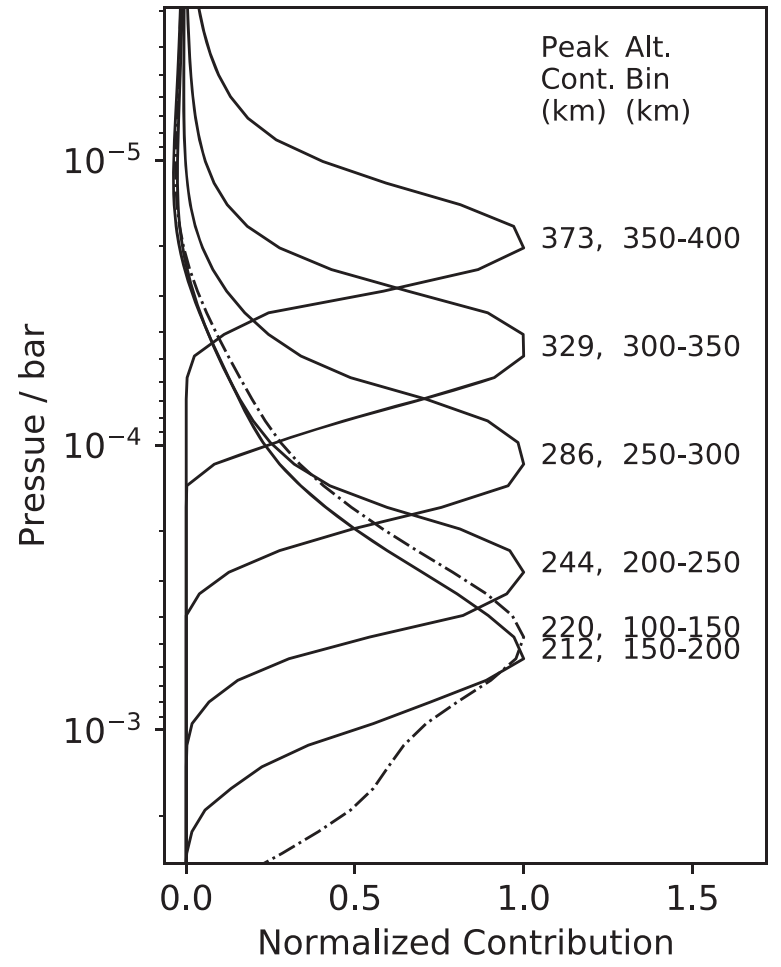

Figure 9. Contribution functions from the $\nu_{8}$ band at $1458 \mathrm{~cm}^{-1}$ achieve maxima very high in the stratosphere due to the high opacity of the molecule at these wavelengths. This is especially noticeable in the contribution function for the altitude bin (shown as the dotted-dashed line for clarity and for which the greatest source of emission in this spectral window is $220 \mathrm{~km}$, much higher than the tangent altitude of the observation of $125 \mathrm{~km}$ ).

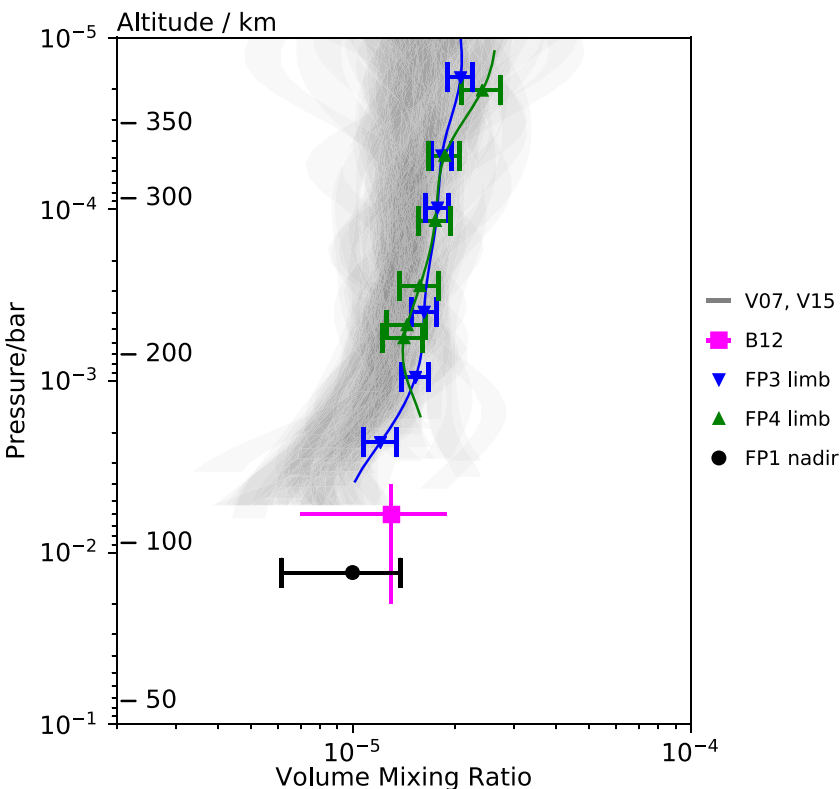

Figure 10. Abundance measured from our FP1 analysis (black) compared to profiles measured from the FP3 (blue) and FP4 (green) analyses. Our vertically resolved profiles are comparable to the measurements made in Vinatier et al. (2007, 2015), V07 and V15 in the figure. Nadir measurements from Bampasidis et al. (2012; pink, B12 in the figure) probe to a slightly higher altitude than that of our measurements.

We have also modeled the $\nu_{8}$ band of ethane, showing that it can be a useful tool in measuring ethane in planetary atmospheres. Future observations of planetary atmospheres may be able to make use of our results by observing both the $\nu_{8}$

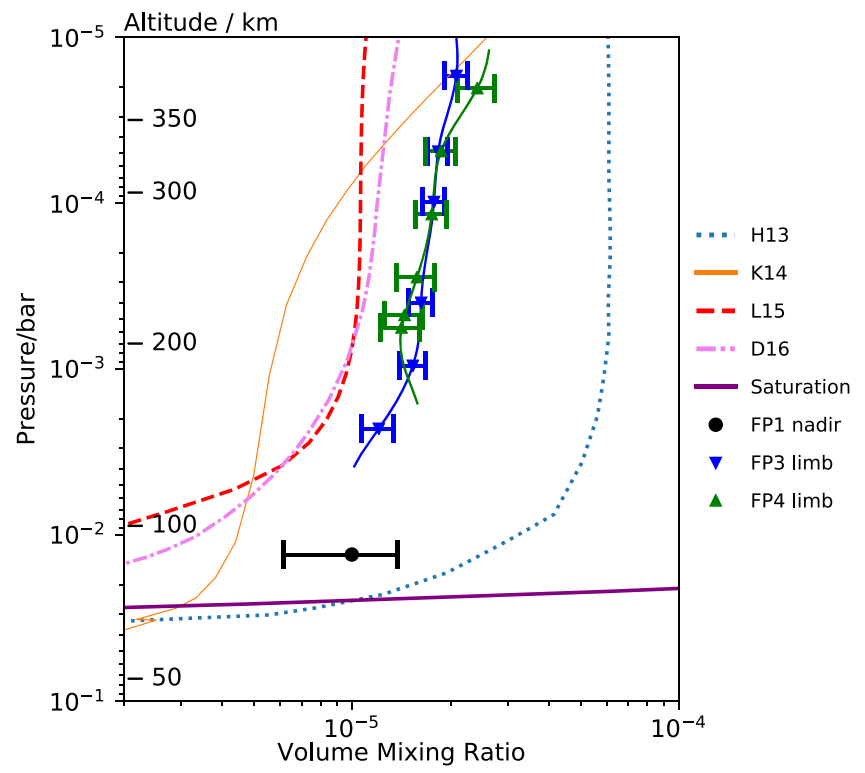

Figure 11. Our measurements at $88 \mathrm{~km}$ made from the FP1 data and above $100 \mathrm{~km}$ for FP3 and FP4 are compared with the predictions from photochemical models. H13 is from Hébrard et al. (2013), K14 is from Krasnopolsky (2014), L15 is from Loison et al. (2015), and D16 is from Dobrijevic et al. (2016). The purple solid line is the saturation abundance of ethane at the temperatures and pressures included in our model. When compared with measurements from mid-infrared observations, our analysis shows that ethane is about as abundant at $88 \mathrm{~km}$ as it is between 100 and $400 \mathrm{~km}$. In contrast, photochemical models predict ethane to be depleted in this region.

band of $\mathrm{C}_{2} \mathrm{H}_{6}$ and the nearby $\nu_{4}$ band of $\mathrm{CH}_{4}$, frequently used as an atmosphere thermometer, simultaneously.

N.A.L. was supported by NASA through the CRESST II cooperative agreement CA 80GSFC17M0002. N.A.L., C.A.N., R.K.A., and F.M.F. were supported by the NASA Cassini Project for the research work reported in this paper. M.S., N.A.T., and P.G.J.I. were funded by the UK Science and Technology Facilities Council.

\section{ORCID iDs}

Conor A. Nixon (1) https://orcid.org/0000-0001-9540-9121 Nicholas Teanby (1) https://orcid.org/0000-0003-3108-5775 Patrick J. G. Irwin @ ittps://orcid.org/0000-0002-6772-384X

\section{References}

Achterberg, R. K., Gierasch, P., Contrath, B., et al. 2014, AAS/DPS Meeting, 46, 102.07

Anderson, C. M., \& Samuelson, R. E. 2011, Icar, 212, 762

Bampasidis, G., Coustenis, A., Achterberg, R. K., et al. 2012, ApJ, 760, 144 Chan, C., Albright, S., Gorius, N., et al. 2015, ExA, 39, 367

Cordier, D., Mousis, O., Lunine, J. I., Lavvas, P., \& Vuitton, V. 2009, ApJL, 707, L128

Cordier, D., Mousis, O., Lunine, J. L., et al. 2012, P\&SS, 61, 99

Cottini, V., Nixon, C., Jennings, D., et al. 2012, Icar, 220, 855

Coustenis, A., Bézard, B., \& Gautier, D. 1989, Icar, 80, 54

Coustenis, A., Jennings, D., Nixon, C., et al. 2010, Icar, 207, 461

Cui, J., Yelle, R., Vuitton, V., et al. 2009, Icar, 200, 581

Danielson, R. E., Caldwell, J. J., \& Larach, D. R. 1973, Icar, 20, 437

di Lauro, C., Lattanzi, F., Brown, L. R., et al. 2012, P\&SS, 60, 93

Dobrijevic, M., Loison, J., Hickson, K., \& Gronoff, G. 2016, Icar, 268, 313

Gillett, F. C., Forrest, W. J., \& Merrill, K. M. 1973, ApJL, 184, L93

Gordon, I., Rothman, L., Hill, C., et al. 2017, JQSRT, 203, 3

Griffith, C. A., Penteado, P., Rannou, P., et al. 2006, Sci, 313, 1620 
Hébrard, E., Dobrijevic, M., Loison, J. C., et al. 2013, A\&A, 552, A132 Irwin, P., Teanby, N., de Kok, R., et al. 2008, JQSRT, 109, 1136 Jacquinet-Husson, N., Armante, R., Scott, N., et al. 2016, JMoSp, 327, 31 Jennings, D. E., Flasar, F. M., Kunde, V. G., et al. 2017, ApOpt, 56, 5274 Krasnopolsky, V. A. 2014, Icar, 236, 83

Lacis, A., \& Oinas, V. 1991, JGR, 96, 9027

Lellouch, E., Bézard, B., Flasar, F., et al. 2014, Icar, 231, 323

LeMouélic, S., Rannou, P., Rodriguez, S., et al. 2012, P\&SS, 60, 86

Li, C., Zhang, X., Gao, P., \& Yung, Y. 2015, ApJL, 803, L19

Loison, J., Hébrard, E., Dobrijevic, M., et al. 2015, Icar, 247, 218

Lombardo, N. A., Nixon, C. A., Achterberg, R. K., et al. 2019, Icar, 317, 454

Magee, B. A., Waite, J. H., Mandt, K. E., et al. 2009, P\&SS, 57, 1895

Mastrogiuseppe, M., Hayes, A., Poggiali, V., et al. 2017, Icar, 300, 203

Mastrogiuseppe, M., Poggiali, V., Hayes, A., et al. 2018, E\&PSL, 496, 89

Moazzen-Ahmadi, N., Oliaee, J. N., Ozier, I., et al. 2015, JQSRT, 151, 123
Niemann, H. B., Atreya, S. K., Demick, J. E., et al. 2010, JGR, 115, E12006

Nixon, C., Achterberg, R., Teanby, N., et al. 2010, FaDi, 147, 95

Nixon, C., Jennings, D., Flaud, J.-M., et al. 2009, P\&SS, 57, 1573

Rengel, M., Sagawa, H., Hartogh, P., et al. 2014, A\&A, 561, A4

Sung, K., Toon, G. C., Mantz, A. W., \& Smith, M. A. H. 2013, Icar, 226, 1499

Sylvestre, M., Teanby, N. A., d'Ollone, J. V., et al. 2019, Icar, in press

Sylvestre, M., Teanby, N. A., Vinatier, S., Lebonnois, S., \& Irwin, P. G. J. 2018, A\&A, 609, A64

Teanby, N., Irwin, P., de Kok, R., et al. 2009, Icar, 202, 620

Teanby, N., Irwin, P., Nixon, C., et al. 2013, P\&SS, 75, 136

Vinatier, S., Bézard, B., Fouchet, T., et al. 2007, Icar, 188, 120

Vinatier, S., Bézard, B., Lebonnois, S., et al. 2015, Icar, 250, 95

Vinatier, S., Rannou, P., Anderson, C. M., et al. 2012, Icar, 219, 5

Vuitton, V., Yelle, R., Klippenstein, S., Hörst, S., \& Lavvas, P. 2018, Icar, 324, 120 